\title{
Télésurveillance et exploitation des télémesures
}

\author{
J.L. Guillermou \\ Eau et force - Parisienne des eaux
}

\section{Le contrôle des comptages}

Un an et demi de travail ont été nécessaires pour fiabiliser le système de comptage de l'eau en gros à Paris et corriger les imprécisions rencontrées. Cette longue étude nous a permis de comprendre les causes d'erreurs rencontrées dues à l'influence de nombreux paramètres dont les effets relatifs ont pu être analysées : la géométrie (paramétrage des appareils), les conditions d'écoulement de l'eau, la dérive dans le temps, les coefficients hydrauliques, l'influence de l'enfoncement des sondes, etc...

Je rends ici hommage à nos Experts qui ont su disséquer les problèmes rencontrés, et nous conseiller sur les meilleures solutions à adopter pour un comptage le plus précis possible, c'est-à-dire dans une fourchette de précision acceptable.

L'ensemble des partenaires de la production et de la distribution de l'eau à Paris est maintenant convenablement outillé pour compter l'eau avec la meilleure précision possible, soit plus ou moins $2 \%$ par appareil et plus ou moins $1 \%$ sur l'ensemble.

$1 \%$, c'est très peu mais aussi beaucoup : cela équivaut à 2,6 millions de $\mathrm{m}^{3}$ par an vendus par la SAGEP aux gestionnaires de la distribution, ou 1,1 million de $\mathrm{m}^{3}$ échangés entre les 2 rives de la Seine.

Il est nécessaire maintenant de s'assurer que la fiabilité du système de comptage perdure dans le temps; en fonction des types d'appareils installés, les moyens de vérification seront différents et les fréquences variables :

- la précision des débitmètres électromagnétiques pourra être contrôlée par passage au banc d'étalonnage mais la dépose/repose de certains gros appareils sera une opération lourde.

- le contrôle des débitmètres à ultrason installés à même la conduite pose encore plus de problèmes; la vérification de la précision sur site n'est pas possible (ou très lourde et très coûteuse) et seule paraît adaptée une vérification de la vraisemblance des indications soit :

\begin{abstract}
- Par mise en place pour essai en parallèle sur les sondes d'une seconde électronique de génération et types différents.

- par contrôle par un capteur électromagnétique à insertion sur la même canalisation (mise en place très facile, donc fréquence de contrôle renforcée).

- Utilisation possible sur certaines petites conduites d'un débitmètre électromagnétique sur manchette en série.

Cependant, on peut noter qu'à tous ces contrôles de précision ou de vraisemblance s'attache une notion de fréquence. Une autre méthode de contrôle consiste à suivre en temps réel non pas chaque appareil individuellement mais la cohérence du système, soit globalement, soit en le découpant en sous systèmes homogènes. C'est ce que je me propose de décrire ici.
\end{abstract}

\section{Le suivi permanent des indicateurs}

Nous disposons à Paris de différents indicateurs dont les informations sont concentrées au dispatching de la SAGEP et relayées sur les deux gestionnaires :

- Comptages généraux et de transfert :

- débits en temps réel (rafraîchis toutes les 3 minutes);

- index de l'intégrateur (sens positif et négatif) ;

- manque de courant, porte de l'armoire ouverte, etc... ;

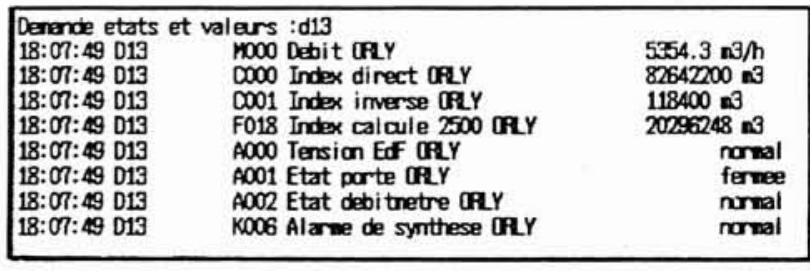

1. Etat d'un poste de comptage.

\section{Remote supervision and measure utilization}

In order to detect problems on the Paris drinkable water supply network, some means were used:

- striking the balance daily, weekly and monthly

- coherence controls of different countings

- flow supervision thanks to a program which compares the real graph and the forcasting graph

- the comparison of the behaviour of a small test ideal network with the one of a sub network. 


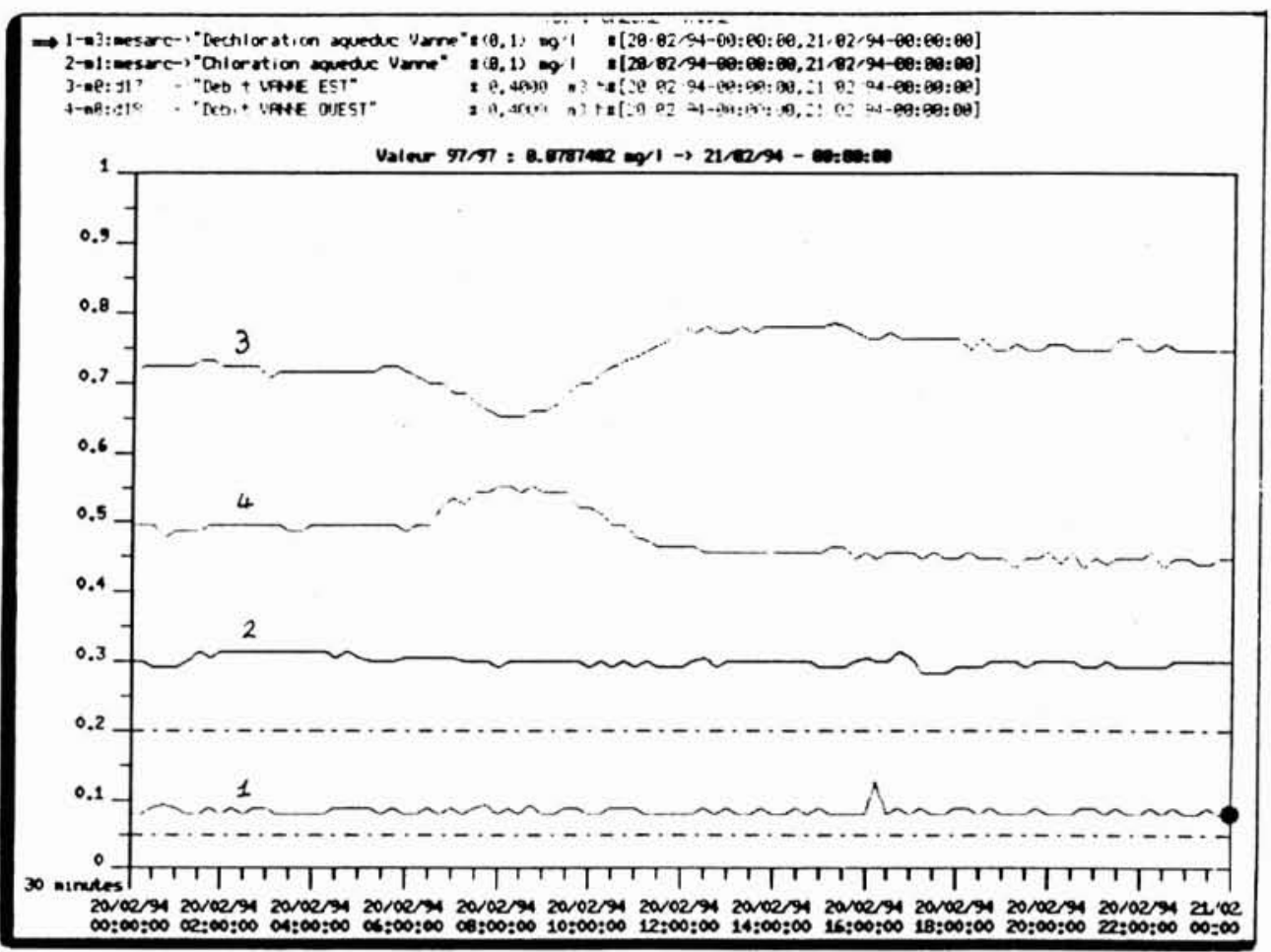

2. Chloration à la mise en distribution.

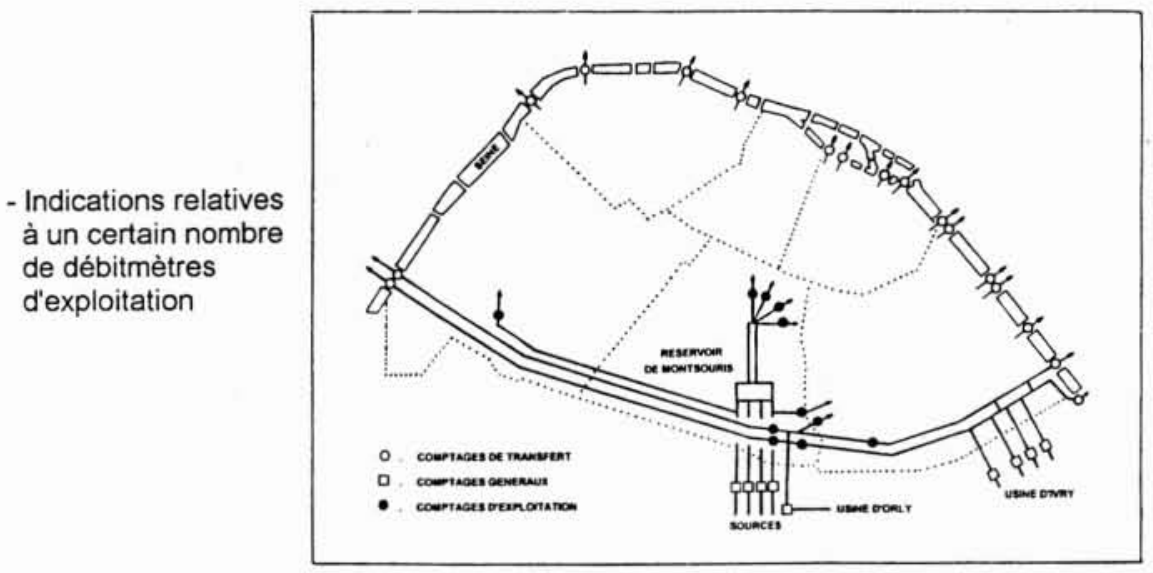

3. Débitmètre principaux- Rive Gauche de Paris.

- Pression en différents points du réseau.

Nous disposerons bientôt également du taux de chlore actif $(\mathrm{Cl} \mathrm{OH})$ en tout point du réseau de distribution (microcapteurs).

La distribution à Paris est divisée en sous réseaux en fonction de l'altitude. Chaque sous réseau a une pression de 4 à 5 bars, du bord de Seine (réseau bas) aux points les plus hauts (Montparnasse/Porte d'Orléans en Rive Gauche, mais aussi Montmartre et autres en Rive Droite).

La Rive Gauche est divisée en 5 sous réseaux d'importance différente :

— Le réseau bas : Le plus difficile à gérer car il est très long et étroit et qu'il y transite une quantité importante d'eau vers la Rivè Droite.

- Les réseaux Panthéon, Vaugirard et Montsouris.

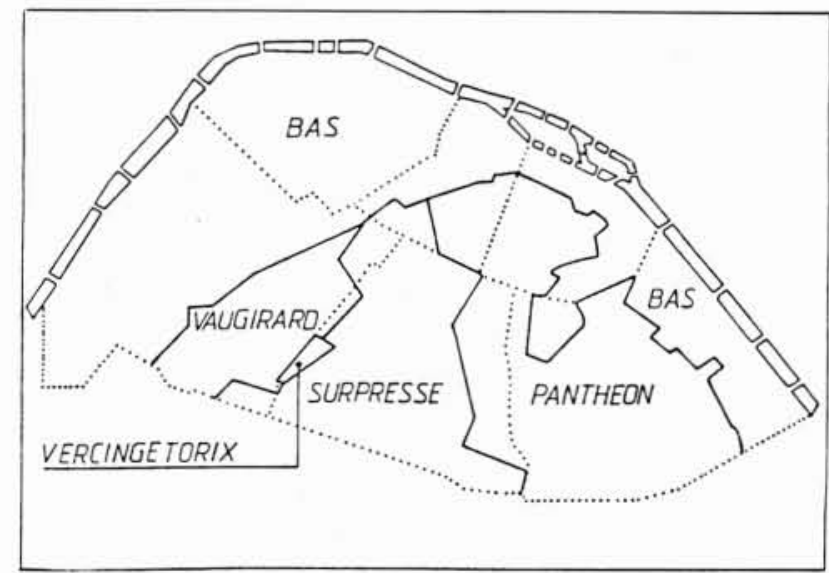

4. Sous réseaux Rive Gauche. 
— Le petit réseau Vercingétorix dont je reparlerai tout à l'heure.

Nous voilà dotés d'un grand nombre d'indicateurs pratiquement en temps réel qu'il nous faut surveiller. Plus les contrôles de cohérence seront réalisés sur de petites unités, plus les contrôles seront fins, plus les anomalies en relatif deviendront importantes et décelables.

\section{Surveillance générale}

Le panorama que l'on observe dépend de l'angle de vue : En grand angle, on aperçoit les tendances, comme les volumes mis en distribution au mois le mois comparés d'une année à l'autre, et les ratios lissés.

\begin{tabular}{|c|c|c|c|c|c|c|}
\hline \multirow{2}{*}{$\begin{array}{c}\text { ANNEE } 1993 \\
\text { ETAT AD }\end{array}$} & \multicolumn{2}{|l|}{ VERSION JLG } & \multirow{2}{*}{$\begin{array}{c}\text { NOV } \\
\text { MESURE }\end{array}$} & \multirow{2}{*}{$\begin{array}{c}\text { DEC } \\
\text { MESURE }\end{array}$} & \multicolumn{2}{|c|}{ TOTAL } \\
\hline & 17-AoQ-94 & & & & MESURE & CORR \\
\hline GENERAUX & ARCUEIL & $\mathbf{A r c}$ & 7049.3 & 8321.6 & 95471.4 & 0.0 \\
\hline RIVE & IVRY & Ivr & 5315.1 & 5279.2 & 55697.9 & 0.0 \\
\hline GAUCHE & ORLY MULTICORDE & Or1 & 4762.0 & 4419.0 & 49842.8 & 0.0 \\
\hline TOTAL & ArctIvr+0r1 & WG & 17126.4 & 18019.8 & \multicolumn{2}{|c|}{$201012.1 \quad 199904.4$} \\
\hline GENERAUX & DHUYS D0+D1 & $\overline{\mathrm{Dh}}$ & 205.9 & 379.1 & 2039.3 & 0.0 \\
\hline RIVE & SAINT-CLOUD & stC & 2516.6 & 2588.9 & 36194.8 & 0.0 \\
\hline DROITE & SAINT-MAUR & StM & 1454.0 & 551.5 & 26869.3 & 0.0 \\
\hline TOTAL & Dh+StC+StM & WD & 4176.5 & 3519.5 & 65103.4 & 64893.3 \\
\hline \multirow{5}{*}{ INTERCOMS } & & F1 & 8.0 & 2.0 & 154.0 & 154.0 \\
\hline & $\begin{array}{l}\text { INTERCOMS RD } \\
\text { INTERCOMS RG }\end{array}$ & ID & 0.0 & 0.0 & -45.0 & -45.0 \\
\hline & INTERCOMS RG & IG & 0.0 & 0.0 & 0.0 & 0.0 \\
\hline & Deversements CEP & DCEP & 0.0 & 38.0 & 196.0 & 196.0 \\
\hline & Deversements SAGEP RD égout & DSDE & 0.0 & 36.0 & 111.0 & 111.0 \\
\hline \multirow{7}{*}{ DEVERSEMENTS } & & DSDN & 4.0 & 0.0 & 1594.3 & 1594.3 \\
\hline & \multirow{2}{*}{$\begin{array}{l}\text { Deversts SAGEP RD DO } \\
\text { Deversements St-Cloud }\end{array}$} & DO & 7.9 & 123.2 & 1047.2 & 1047.2 \\
\hline & & DSC & 0.0 & 0.0 & 72.0 & 72.0 \\
\hline & Deversements SPE & DSP & 10.0 & 0.0 & 28.0 & 28.0 \\
\hline & Deversements SAGEP RG & DSG & 0.0 & 0.0 & 205.0 & 205.0 \\
\hline & MARNAGES RD & MRD & -24.0 & 73.0 & 45.0 & 45.0 \\
\hline & MARNAGES RG & MRG & 56.0 & 19.0 & 31.0 & 31.0 \\
\hline PONTS & correction: & $\mathbf{P}$ & 10185.9 & 10985.5 & 116483.9 & 116512.1 \\
\hline \multirow{3}{*}{$\begin{array}{l}\text { VOLUMES } \\
\text { FOURNIS } \\
\text { PAR LA SAGEP }\end{array}$} & \multirow{3}{*}{$\begin{array}{lc}\text { TOTAL LIVRE } & \text { WG+WD+F1+ID+IG-DSDE.N-D0-DSG+DSC } \\
\text { VOL LIVRE CEP } & \text { WD+P+F1+ID-DSDE.N-DO+DSC } \\
\text { VOL LIVRE SPE } & \text { WG-P+IG-DSG }\end{array}$} & $\overline{\text { TD }}$ & 21299.0 & 21382.1 & 263339.0 & 262021.2 \\
\hline & & RD & 14358.5 & 14347.8 & 179015.8 & 178833.9 \\
\hline & & RG & 6940.5 & 7034.3 & 84323.2 & 83187.3 \\
\hline & FACTURB RD & FD & 14093 & 14082 & 175824 & 175642 \\
\hline & RG- $(94+121)$ & FG & 6726 & 6819 & 81743 & 80607 \\
\hline $\begin{array}{l}\text { VOLUMES MIS EN } \\
\text { DISTRIBUTION }\end{array}$ & $\begin{array}{c}\text { TD + MARNAGES } \\
\text { RG + MARNAGES RG }\end{array}$ & $\begin{array}{l}\text { TDD } \\
\text { RGD }\end{array}$ & $\begin{array}{r}21331.0 \\
6996.5\end{array}$ & $\begin{array}{r}21474.1 \\
7053.3\end{array}$ & $\begin{array}{r}263415.0 \\
84354.2\end{array}$ & $\begin{array}{r}262097.2 \\
83218.3\end{array}$ \\
\hline PAR LES GESTIONN. & RD + MARKAGES RD & RDD & 14334.5 & 14420.8 & 179060.8 & 178878.9 \\
\hline & RATIO DISTRI & W & $32.80 z$ & 32.852 & 32.022 & 31.752 \\
\hline & Rappel Dist.RG93 & RGD & 6996.5 & 7053.3 & 84354.2 & 83218.3 \\
\hline & AVEC CORR DES PONTS & & 7041.0 & 6833.0 & 86018.0 & 86018.0 \\
\hline & ORLY = D.13; PONTS $-2,85 Z$ & & 6965.0 & 6701.0 & 86013.0 & 86013.0 \\
\hline & Evolution $93 / 92$ & & $-0.63 z$ & 3.227 & $-1.93 z$ & -3.252 \\
\hline & Rappe1 Dist.RD93 & RDD & 14334.5 & 14420.8 & 179060.8 & 178878.9 \\
\hline & AVEC CORR DES PONTS & & 14565.0 & 14418.0 & 185502.0 & 185502.0 \\
\hline & Evolution $93 / 92$ & & $-1.58 z$ & 0.022 & $-3.47 z$ & -3.572 \\
\hline & Rappe1 Total 93 & TDD & 21331.0 & 21474.1 & 263415.0 & 262097.2 \\
\hline & AVEC CORR DES PONTS & & 21606.0 & 21251.0 & 271520.0 & 271520.0 \\
\hline & ORLY $=D^{\prime} 13 ;$ PONTS $-2,857$ & & $\begin{array}{r}22631.0 \\
-1.272\end{array}$ & $\begin{array}{r}22572.0 \\
1.052\end{array}$ & $\begin{array}{r}283786.0 \\
-2.997\end{array}$ & $\begin{array}{r}283786.0 \\
-3.472\end{array}$ \\
\hline
\end{tabular}

5. Suivi mensuel de la mise en distribution (extrait). 
Avec un objectif normal, contrôle hebdomadaire, jour après jour, toujours sous forme de bilan, mais plus fin puisqu'on examine les données compteur par compteur :

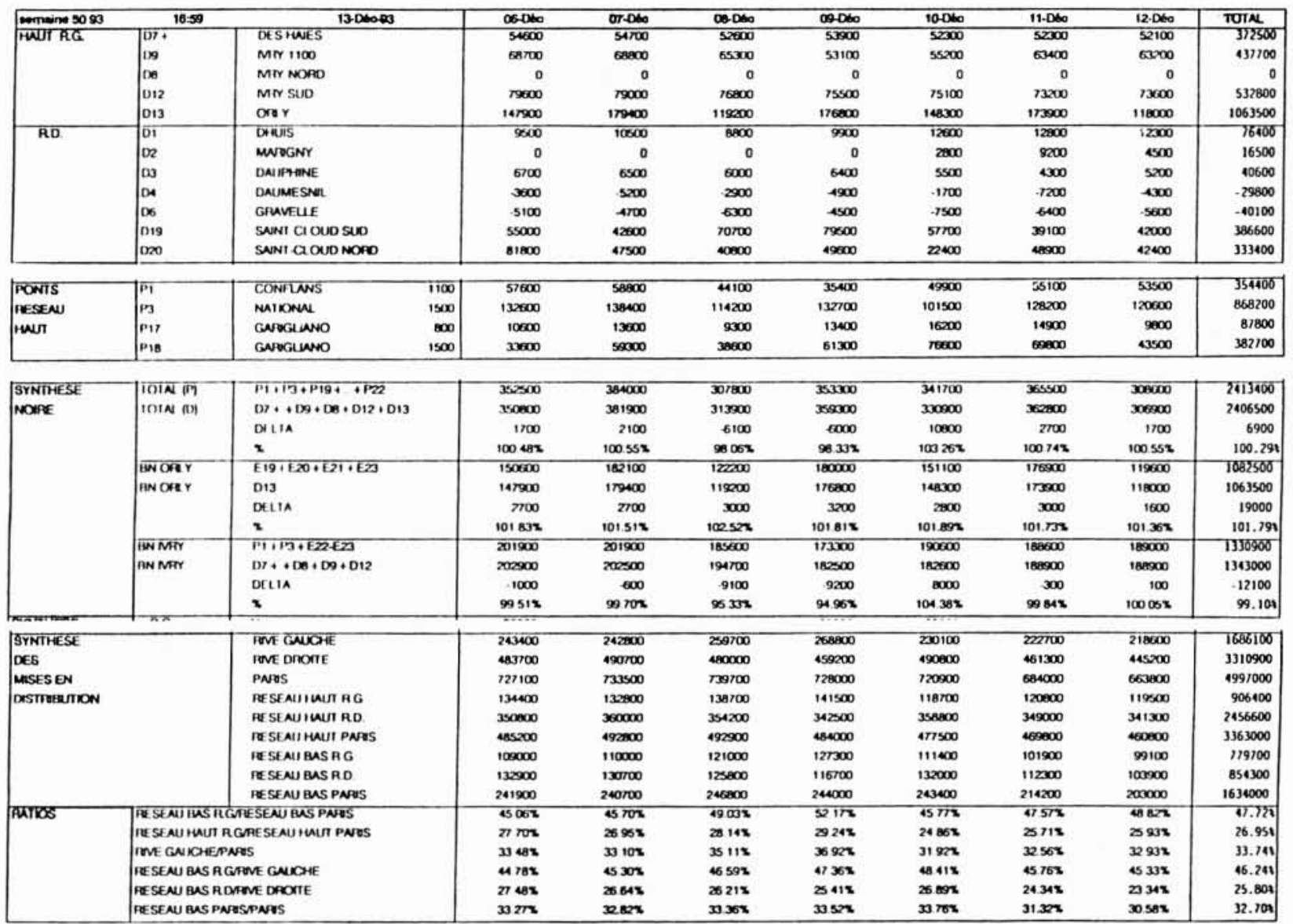

6. Bilan hebdomadaire des comptage périphériques et de transferts : extraits

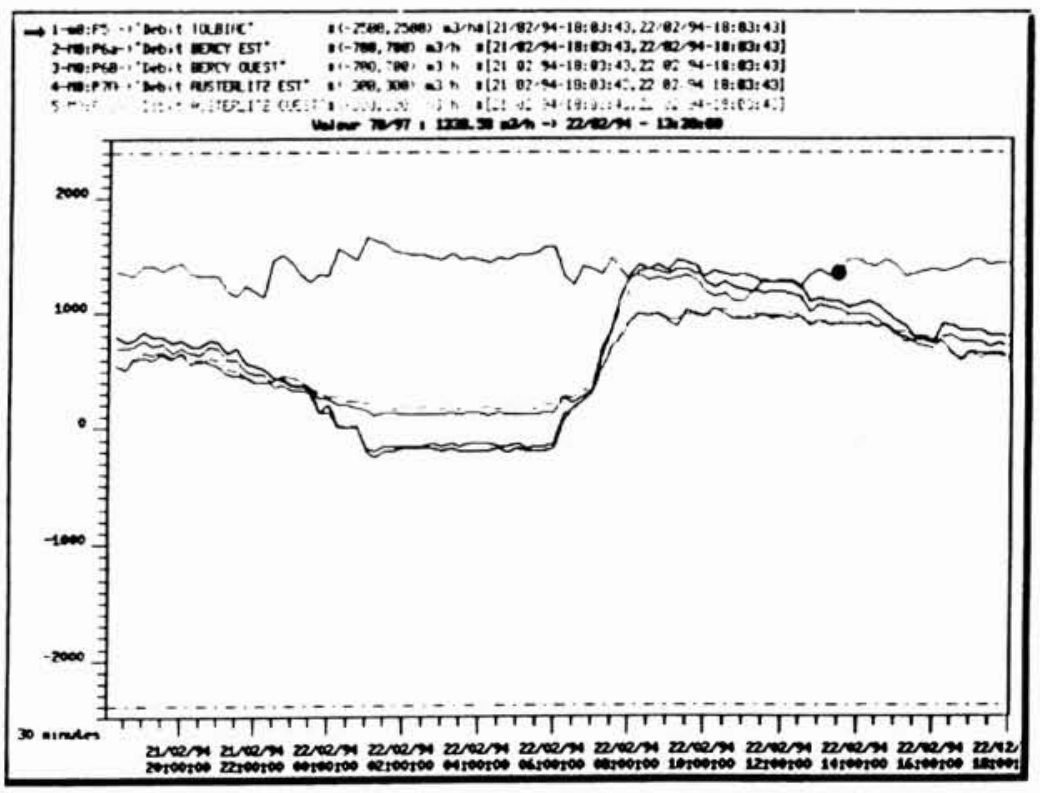

Le zoom permet de descendre à l'intérieur de la journée, par tranches, de 6 heures, où même d'une heure. On pourra également travailler en débit et non plus en volume pour interpréter les phénomènes.

7. Suivi des débits de cinq comptages de transfert. 


\section{Contrôle de cohérence}

A l'intérieur même des bilans sont réalisés des contrôles de cohérence des indicateurs.

A partir de l'ensemble de nos divers comptages généraux, de transfert, d'exploitation, rapprochons nous un peu :

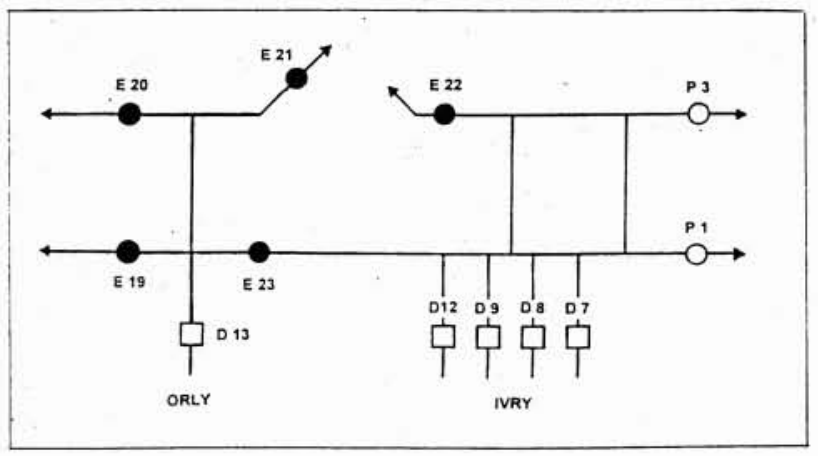

\section{Boîte Globale - Réseau haut.}

Le shéma 8 simplifié montre que l'ensemble des eaux en provenance des usines d'Orly et Ivry est véhiculé par des conduites de grand diamètre ; il est à nouveau compté par un certain nombre de comptages d'exploitation ou de transfert. La somme algébrique de ce qui entre et de ce qui sort de cette «boîte " doit être nulle (à la précision de chacun des compteurs près); toute anomalie est signe de la défaillance d'un des compteurs ou d'une fuite à l'intérieur de la boîte. Si on se rapproche encore un peu, on constate que deux petites boîtes sont contenue dans la grande :

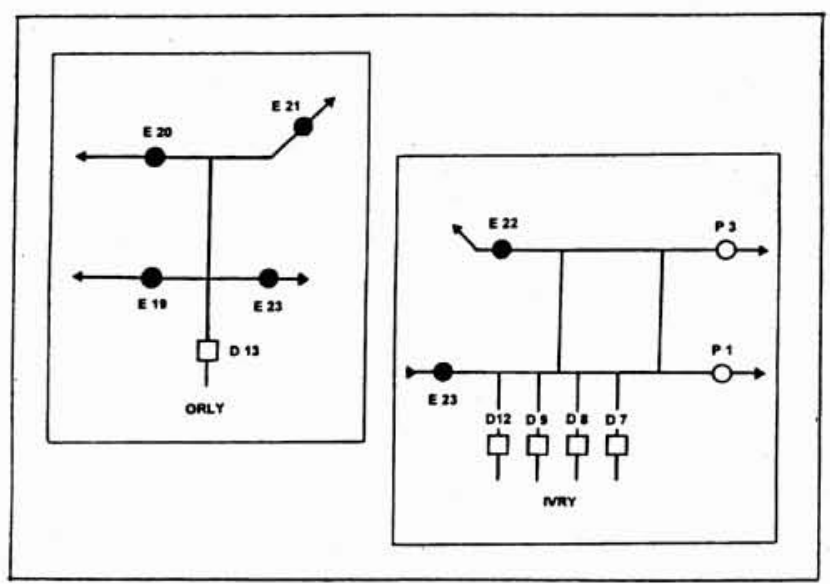

\section{Demi-boîtes - Réseau haut.}

Ces bilans sont observés chaque semaine, pour contrôle à titre historique, en volume. Bien évidemment un contrôle de boîte peut être réalisé en débit en temps réel :

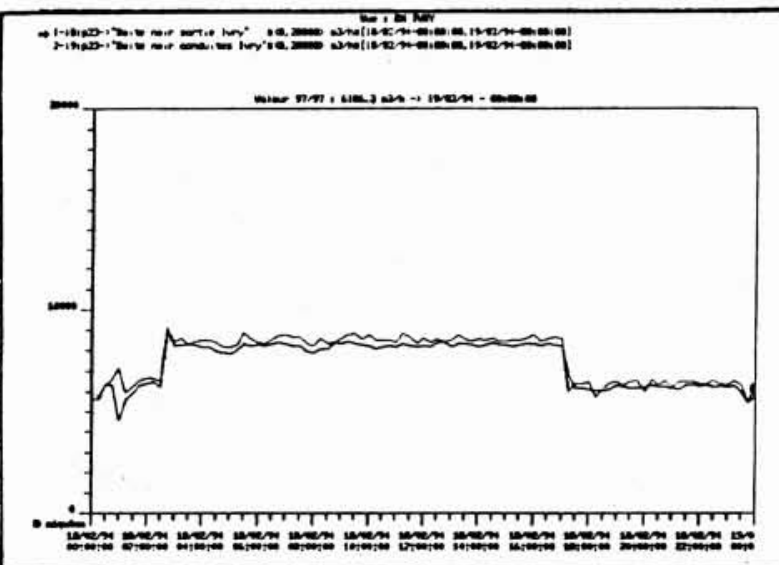

10. Contrôle de boîte en temps réel.

Les moyens de contrôle en débit ne sont encore pas tous créés ; ils doivent être couplés à des systèmes d'alarme dès que l'écart entre les deux courbes est supérieur à une limite fixée.

\section{Surveillance des débits}

L'alimentation de Paris en eau potable est assurée à partir de sources depuis le siècle dernier qui assurent encore plus de la moitié des besoins de la Capitale, et de trois usines traitant les eaux de la Seine et de la Marne.

Si les sources ont des débits assez réguliers, les fournitures par les usines sont fonction des règles d'exploitation fixées par la SAGEP; les débits et volumes correspondants sont assez variables.

De la même façon, les volumes transitant par les ponts et les compteurs d'exploitation varient en fonction des contrainte de l'exploitation, de biefs hors service pour travaux par exemple.

Par contre, chacun des sous réseaux a un comportement spécifique, assez reproductible d'une journée à l'autre en semaine; les samedi se ressemblent tous aussi, ainsi que les dimanche. Seuls sont atypiques les périodes de congés, les jours fériés avec ou sans pont.

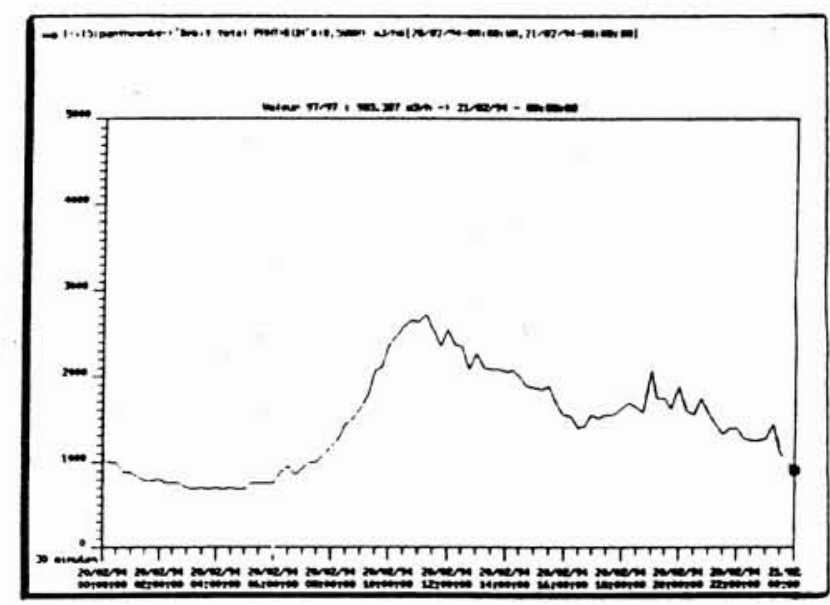

11. Débit journalier d'un sous réseau. 


\section{TÉLÉSURVEILLANCE ET EXPLOITATION DES TÉLÉMESURES}

Nous avons donc pu envisager de "prévoir " la courbe de débit d'une journée et la comparer à la courbe réelle ; tout écart est signe d'anomalie à traiter rapidement dès que le système nous a donné l'alarme.

Le laboratoire d'Informatique Avancée de Compiègne (LIAC) avec la collaboration du CA2I, a mise en place pour la société Parisienne des Eaux, un système de détection de fuites par comparaison du débit mesuré en temps réel et de la courbe du débit prévu la veille pour le lendemain. Ce système, nommé DETECTOR, est composé de 4 modules principaux :

- Acquisition : une carte Applicom 4000 acquiert toutes les 5 minutes les mesures de débit du réseau transmises via un modem. Le module récupère ces mesures, calcule le débit moyen tous les quarts d'heure et l'affiche sur l'écran graphiquement.

- Prévision : tous les jours, à 4 heures du matin (où le débit atteint son minimum), le module de prévision est activé pour calculer la courbe de débit prévu. Plusieurs réseaux de neurones artificiels (RNA), qui ont été construits à partir des données historiques, sont utilisées pour réaliser cette prévision. Les débits mesurés des jours précédents et le type de jour (jour de la semaine, jour férié) sont pris en compte dans le calcul. La prévision est affichée sur l'écran graphique, en même temps que le débit mesuré. Ceci permet à l'opérateur de suivre en permanence l'évolution du débit mesuré par rapport à la courbe prévue de la journée.

- Alarme : ce module détecte une situation de surconsommation anormale, et déclenche une alarme si elle se prolonge. Une alarme levée peut être ensuite validée (situation de fuites) ou rejetée (surconsommation inhabituelle). - Visualisation: ce module permet à l'opérateur de visualiser, selon son choix, des courbes de débit mesuré et prévu sur des données archivées.

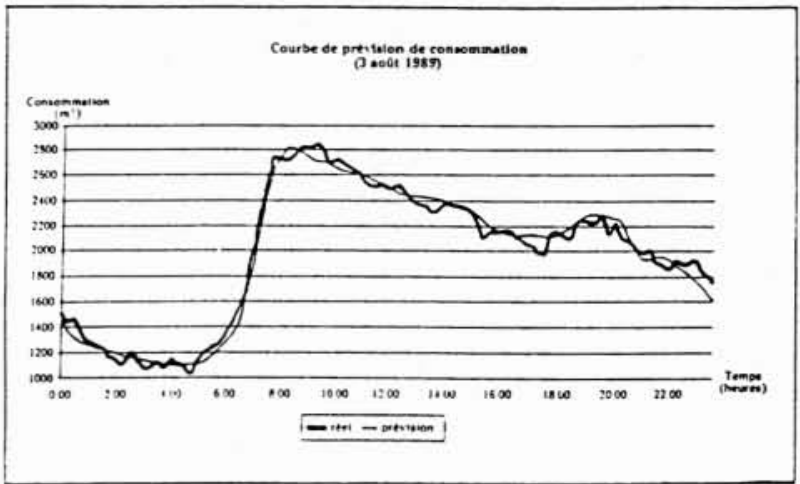

12. Courbe prévue et réelle.
Ce système de prévision et d'alerte est maintenant opérationnel sur le réseau Montsouris; il nous a permis de déceler rapidement une fuite supérieure à $1000 \mathrm{~m}^{3} / \mathrm{h}$ qui n'aurait été mise en évidence que quelques heures plus tard puisque l'eau s'écoule directement à l'égout.

Ce système doit être étendu courant 1994 à l'ensemble des sous réseaux.

Par la suite, un autre système développé en parallèle pourra y être couplé ; plus lourd dans son utilisation, donc à ne mettre en œuvre qu'en cas d'anomalie décelée, il permettra de localiser l'incident :

A partir du système d'information géographique (APIC) est créé un fichier transféré sur le programme de calcul de réseaux maillés (PICCOLO), puis un modèle de réseau simplifié.

Plusieurs débitmètres sont installés sur des tronçons caractéristiques du réseau, dont les indications sont transmises par ligne téléphonique au calculateur.

Le réseau ayant fait l'objet d'un calage préalable, le calculateur détermine, en fonction du débit entrant, le débit attendu sur chacun des débitmètres et le compare aux débits réels. La séquence suivante du programme permet, en fonction des divers écarts constatés, de localiser sur le réseau le lieu probable de l'incident.

Testé avec succès sur le sous réseau Vaugirard, ce système de localisation sera mis en œuvre sur chacun des sous réseaux en complément de DETECTOR.

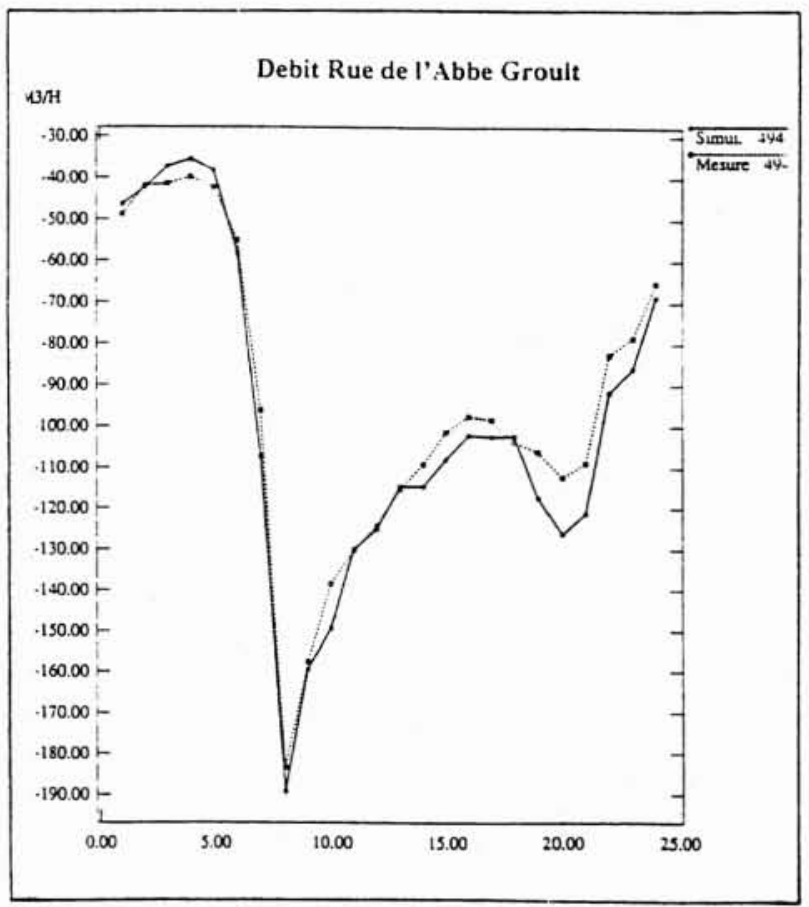

13. Suivi d'un débitmètre en état normal. 


\section{Le baromètre}

Un baromètre ne sert pas qu'à mesurer une pression atmosphérique, mais aussi des tendances ; si la pression monte ou baisse, on peut prévoir l'évolution du temps.

Nous avons créé notre propre baromètre, le réseau Vercingétorix :

- 1 seule entrée, 1 débitmètre.

- 129 compteurs chez les clients, neufs, avec tête émettrice.

- $2,8 \mathrm{~km}$ de réseau visitable sans fuite (vérifié très régulièrement).

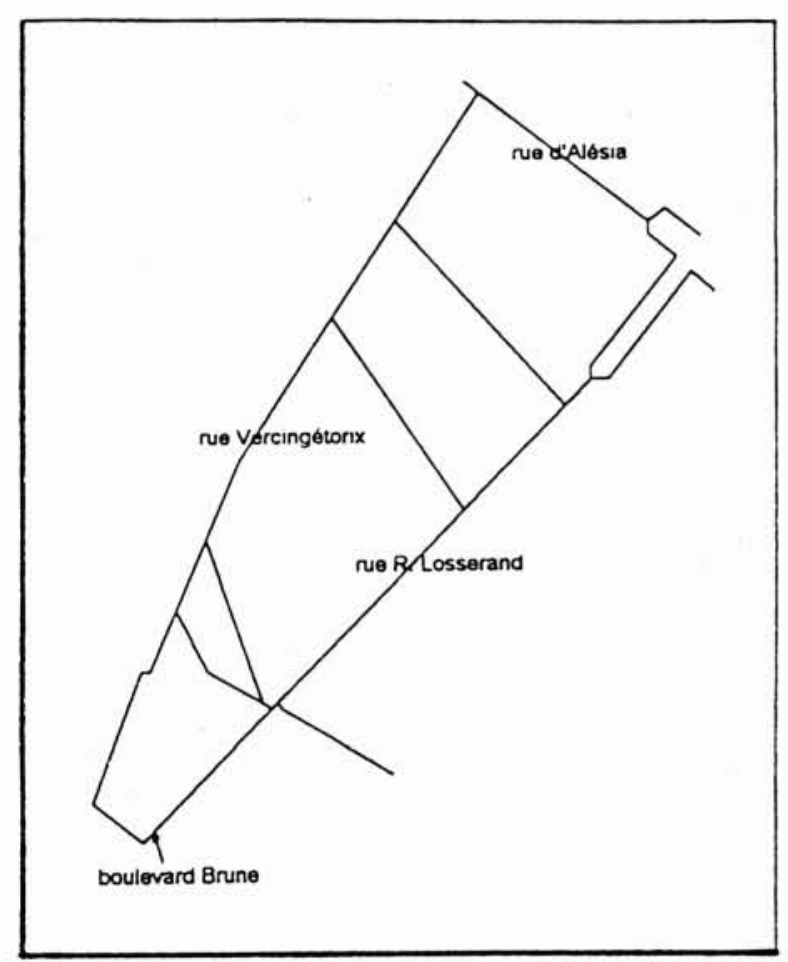

\section{Le réseau Vercingétorix.}

- L'ensemble des indications du débitmètre de tête et des 129 compteurs sont rapatriés par fil toutes les 6 minutes sur un micro PC qui les stocke.

— L'exploitation de ces données nous a déjà permis de faire plusieurs constatations :

- Le rendement d'un réseau où les pertes sont circonscrites aux seuls compteurs des clients avoisine $97 \%$, si les compteurs sont neufs mais non recalibrés (une vingtaine d'entre eux - parmi les plus gros- sont surcalibrés de 2 diamètres).

- Comme on pouvait s'y attendre en fonction de la courbe de fonctionnement classique des compteurs des clients, la courbe du rendement suit celles des débits globaux, de $99 \%$ en débit de pointe à $85 \%$ en débit de nuit.

- Par ailleurs, nous comparons chaque semaine le volume entrant dans ce sous réseau très surveillé, au volume mis en distribution sur l'ensemble de la Rive gauche. Ce pourcentage est stable autour de $0,73 \%$.
Toute variation importante de ce pourcentage serait signe d'une anomalie sur la distribution de la Rive Gauche. Cependant, et pour l'instant encore, la précision du baromètre est faible car une anomalie de $1000 \mathrm{~m}^{3} / \mathrm{h}$ sur le réseau glonal n'entraîne qu'une variation de $0,003 \%$ du baromètre.

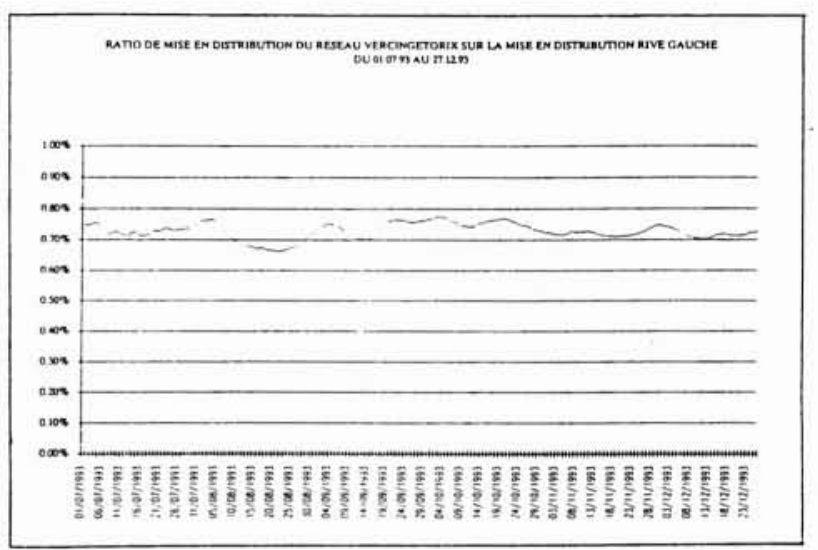

15. Baromètre Vercingétorix.

Dès que l'ensemble de comptages d'entrée des sous réseaux aura pu être fiabilisé (1994 en principe), la fonction baromètre sera appliquée à chacun de ces sous réseaux et notre analyse deviendra beaucoup plus fine.

\section{Conclusion}

La distribution de l'eau potable à Paris est vraiment très particulière :

- 2 millions d'habitants, mais 4 millions de consommateurs le jour ;

- un réseau assez ancien (une bonne partie date du siècle dernier) mais placé à $95 \%$ en galerie technique (les égouts de Paris) donc visitable ;

- un nombre de branchements réduit $(90000)$ pour une consommation de 240 millions de $\mathrm{m}^{3}$ par an ;

— une capacité de stockage supérieure à la journée de consommation moyenne ;

- la distribution confiée à deux gestionnaires chargés chacun d'une zone géographique de la Capitale.

Confrontés à cette situation inhabituelle en 1985, les deux gestionnaires ont réussi, grâce à leurs efforts, à porter en neuf ans le rendement du réseau de $78 \%$ à plus de $90 \%$.

Cependant, ces efforts doivent être toujours soutenus en particulier sur deux points :

à $90 \%$, le rendement ne me paraît encore pas près de son asymptote, même si chaque point d'accroissement sera de plus en plus dur à gagner.

Le rendement d'un réseau est fragile et l'on doit veiller jour après jour à ce que la pente de sa courbe reste positive car les points perdus sont difficiles à rattraper.

Nous avons maintenant beaucoup d'outils adaptés à cette veille.

Cependant, notre connaissance et nos moyens ne sont jamais complets ; il restera encore et encore à inventer des outils afin de mieux contrôler, analyser, comprendre. 